\title{
Computational and experimental study on dynamic instability of extended bistable carbon/epoxy booms subjected to bending
}

\author{
C. $\mathrm{Wu}^{1}, \mathrm{~A}$. Viquerat ${ }^{2, *}$ \\ Faculty of Engineering and Physical Sciences, University of Surrey, GU2 7XH, United Kingdom
}

\begin{abstract}
Bistable reeled composite booms (BRCs) constructed from braided carbon/epoxy plies are suitable candidates for use as extendible booms or as elements of large deployable space structures. However, without modification, BRCs have an open section which limits their torsional stiffness, and makes them prone to collapse under low bending moments. In this study a "roll-up" deployable photovoltaic (PV) solar array with two side-by-side extendible BRCs is used as a case study to numerically analyse the dynamic behaviours of BRCs on spacecraft undergoing rotational manoeuvres. The BRCs have rotational accelerations applied to their roots to simulate the effect of being attached to a manoeuvring spacecraft. Budiansky-Hutchinson criterion is used to define an instability failure point based on a change in cross-sectional shape. This was used to estimate the maximum angular acceleration. While it is extremely difficult to replicate the behaviour of a large flexible lightweight structure in microgravity on the ground, an experiment to determine the point of collapse of BRCs under gravity were used to verify the simulation results.
\end{abstract}

Keywords:

Bistable composites, Dynamic stability, Numerical analysis, Solar array

\section{Introduction}

In their extended stable state, BRCs look like carpenter's tapes (see Fig. 1 (b)), however, they can also be stable in a coiled configuration as illustrated in Fig. 1 (a). BRCs constructed from lightweight braided carbon/epoxy plies having a naturally high packing efficiency makes them excellent candidates for space applications that require structures to be very large in space but as small as possible when launched.

As a result of their potential applications in space, many researchers have put intensive efforts into studying bistable cylindrical shells $[1,2,3,4,5,6]$. Iqbal $[1,2]$ generated an approximate equation of strain energy due to bending and stretching in terms of transverse and longitudinal curvatures for a composite slit tube under pure bending. Galletly [4] developed this further using both beam or shell models with consideration of twisting terms, and

\footnotetext{
${ }^{*}$ Corresponding author

Email address: a.viquerat@surrey.ac.uk (A. Viquerat)

${ }^{1} \mathrm{PhD}$ Candidate

${ }^{2}$ Lecturer
} 
the second equilibrium points were successfully found for different laminated shells. Guest [5] assumed that the mid-surface of the shell did not stretch and the bending experienced by the shell was uniform everywhere to develop a simplified analytical model to predict the bistability and coiled curvature for bistable shells. Fernandez [6] manufactured 'bistable over the whole length' booms for gossamer structures - solar sailing and spacecraft deorbiting applications. However, without modification (interlocking teeth or a "zipper" mechanism), BRCs have low torsional stiffness due to their open cross-sections, which makes them prone to collapse under low bending moments. Thus, the dynamic stability of BRC's subjected to bending is a topic of interest to study in order to understand the scalability of large BRCs for space applications.

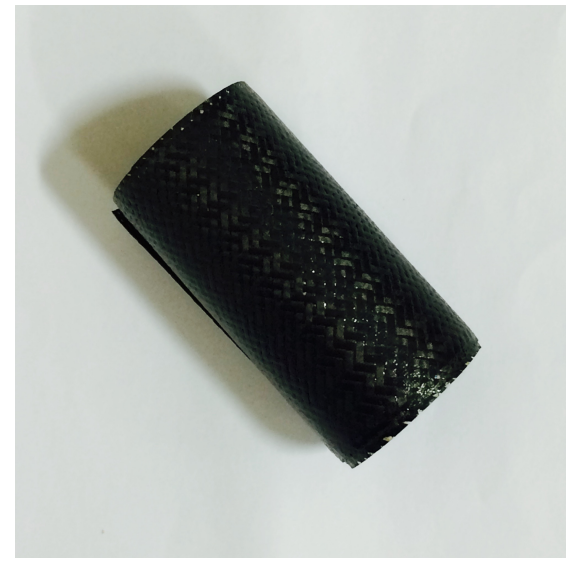

(a) Coiled stable state

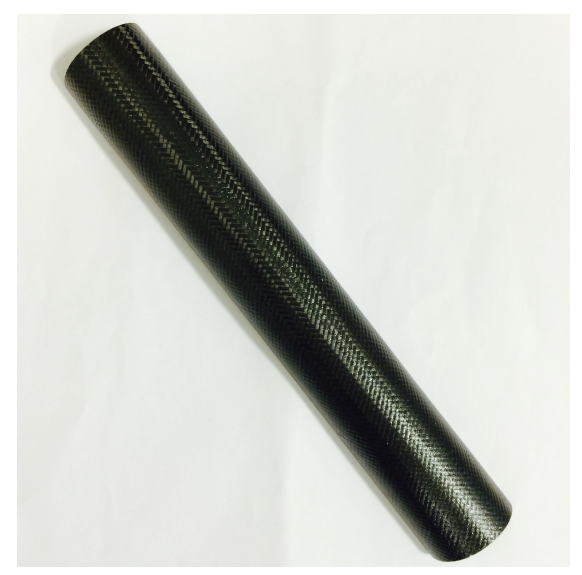

(b) Extended stable state

Fig. 1: Carbon/epoxy BRC tubes in two stable configurations [7].

The dynamic behaviour of composite shell structures subjected to various loads has been studied by a number of researchers $[8,9,10,11,12,13]$. Modi [8] presented more than two hundred references on rigid bodies attached with flexible appendages, with a particular focus on the behaviour of flexible bodies built into a moving base, such as spacecraft antennae, flexible satellites, and helicopter rotor blades. Benedetti [9] derived equations of motion for a uniform Euler-Bernoulli beam with a constant circular cross-section subjected to specified translational and rotational accelerations. However, Benedetti's work analysed the dynamic responses of symmetric closed cross-sections, making the work difficult to apply to opensection booms like the self-storing tubular extensible module (STEM) booms often used on satellites [10].

For practical applications, space-based imaging satellites normally require agility in manoeuvring between image collection attitudes $[14,15,16,17,18,19,20]$, because the time of each collection region in the sensor field-of-view is quite limited, the productivity of imaging satellites directly depends on the manoeuvring agility. Previous study of the natural frequency optimisation of the "roll-up" PV solar array [21, 7] demonstrated that the first vibration mode of the cantilevered solar array involves an up-down cantilever motion, causing the solar array to be prone to bending once it is excited, which makes the behaviour of the solar array subjected to bending of interest to study.

The work presented here is applicable to individual extended BRCs (attached as a can- 
tilever to a host spacecraft), as well as a deployed "roll-up" PV solar array constructed with two parallel and symmetric carbon fibre reinforced polymer BRCs with a flexible PV cell-covered blanket in-between (shown in Fig. 2 (a)).

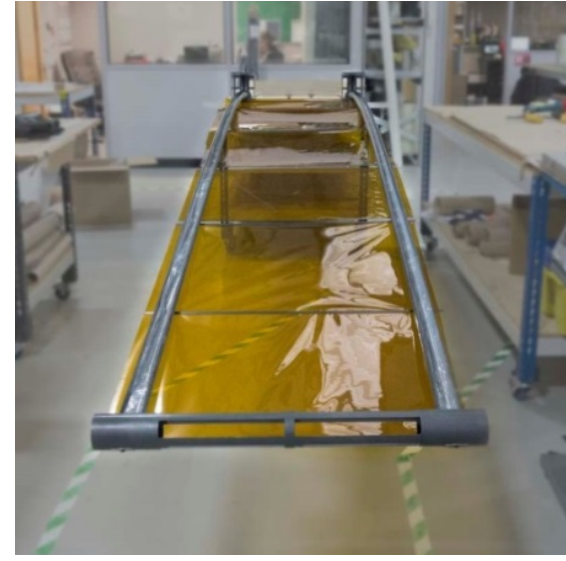

(a) Deployable "roll-up" PV solar array developed for DeployTech

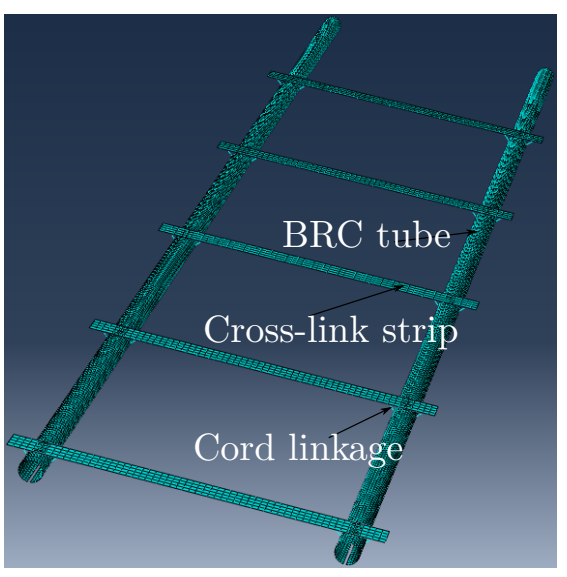

(b) Assembly of a deployable "roll-up" solar array with no PV blanket in ABAQUS

Fig. 2: Deployed "roll-up" PV solar array [21].

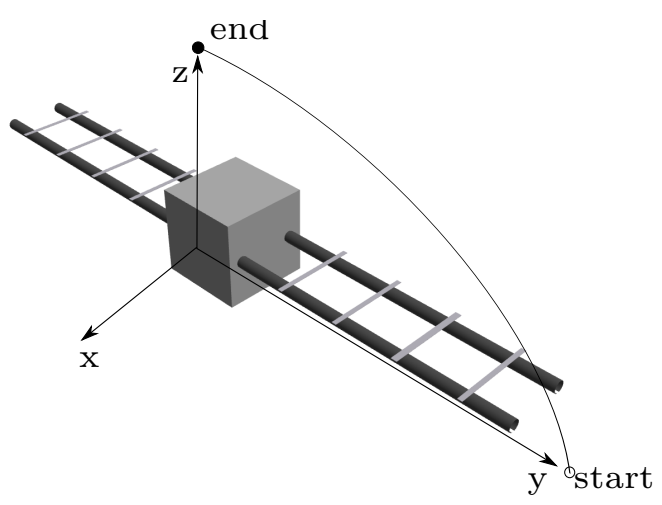

Fig. 3: A simple space-based imaging satellite coupled with symmetrically deployed solar panels. $x$ axis is the axis about which the rotational spacecraft manoeuvre from start to end is investigated. The $z$ axis is the axis along which a translational manoeuvre is computationally and experimentally studied in this article by introducing a linear acceleration.

This paper presents an analysis of the dynamic response of cantilevered single booms and a complete solar array attached to a manoeuvring satellite that is subjected to a specified rotational acceleration or a specified linear acceleration along the $z$ axis (seen in Fig. 3). The formulation of the Finite Element (FE) model of both a single boom and an entire array (with no PV membrane see Fig. 2 (b)) in ABAQUS [22] are described. The stability performances of booms under both same-sense and opposite-sense bending induced from the rotational and translational accelerations are studied. The Budiansky-Hutchinson criterion developed for shell structures is used to estimate the maximum sustainable angular acceleration that BRCs can withstand before becoming unstable. The feasibility of analysing one single BRC 
instead of the whole solar array structure is validated by modelling a deployable "roll-up" solar array and comparing the results to those of a single BRC. An experimental verification was undertaken to validate the simulation results, followed by an analysis of the limits of scale for the use of BRCs on the satellite undergoing a conventional spacecraft manoeuvre and concluding remarks.

\section{Finite Element Model Formulation}

An FE approach was used for the vibration modal analysis of deployed BRC booms in a number of previous papers $[23,24,7,21]$ to capture the lowest frequency vibration mode, which tends to involve a simple up-down "cantilever" motion [25] in which the boom tip locus lies only within the $Y-Z$ (see Fig. 4) plane. In this section, a cantilever single boom model and a complete solar array model without the cell-covered PV blanket in between are created to simulate the dynamic stability performance during the specified manoeuvres.

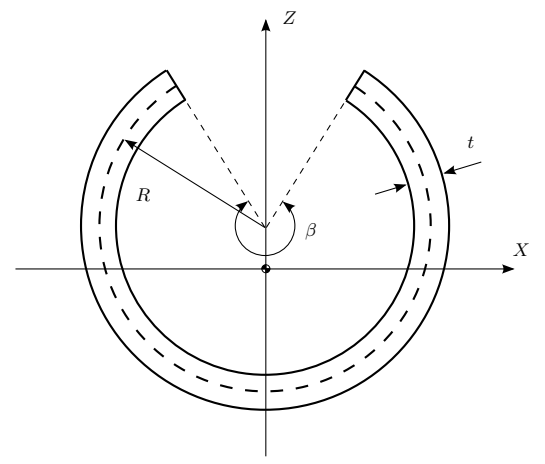

Fig. 4: BRC boom cross-section. $\beta$ is the included angle, $t$ is the tube thickness, $R$ denotes the initial radius measured to the midplane of the tube wall, and $X$ and $Z$ are the axes of the cross-section [21].

Braided fibre is often used in the production of BRCs because it can be used to produce very long slender composites with non-zero fibre angles with no discontinuities along the tube length. It is sometimes also possible to adjust the braided fibre angles manually before impregnation with resin, allowing for the production of BRCs with customised properties, or even properties which vary along its length [6]. However, for this analysis it is assumed that all BRCs have constant material and structural properties along the their length. All braid angles are measured from the boom longitudinal $(Y)$ axis shown in Fig. 4. A single braid ply is taken as two co-located unidirectional (UD) plies, and each of them takes half of the properties.

\subsection{Single Boom Model}

A three dimensional shell model was created in ABAQUS [22] to compute the vibration characteristics of fixed-free single booms by using a linear perturbation approach [21]. Classical laminate theory (CLT) [26] was employed to capture the mechanical properties of the booms. An approximate global shell element (S4R) size of $0.005 \mathrm{~m}^{2}$ and ten elements per wavelength around the circumference (assuming a single wavelength is determined by the circumference of the boom for the first mode [21]) were used to mesh the model. 


\subsection{Solar Array Model}

The FE model of a BRC-based deployed solar array (without the PV blanket) developed in a previous paper [21] is again created to analyse its dynamic stability. $80 \mathrm{~mm}$ wide and $1200 \mathrm{~mm}$ long discrete rigid shell planar strips with a mass $0.1 \mathrm{~kg}$ of each were used to rigidly connect the two parallel BRC booms, spacing at $1 \mathrm{~m}$ intervals along the array length. High tensile elastic $\left(\rho=1000 \mathrm{~kg} / \mathrm{m}^{3}, E=20 \mathrm{GPa}, \nu=0.3\right)$ cord linkages with a radius of $1 \mathrm{~mm}$ connect the strips to the booms. The cross-link strips (seen in Fig. 2 (b)) are constructed using four-node three dimensional bilinear rigid quadrilateral elements (R3D) [22]. The cord linkages are modelled as beam elements (B31). Each BRC is meshed with an approximate global element size of $0.005 \mathrm{~m}^{2}$ and ten approximately square S4R shell elements per wavelength. Carbon/epoxy with the material properties given in Tab. 1 was used and implemented in the boom model to compute the lamina stiffness.

Tab. 1: Material properties of half-carbon/epoxy-braid and UD plies [6]

\begin{tabular}{lllllllll}
\hline \hline Materials & $\begin{array}{l}E_{1} \\
(\mathrm{GPa})\end{array}$ & $\begin{array}{l}E_{2} \\
(\mathrm{GPa})\end{array}$ & $\nu_{12}$ & $\begin{array}{l}G_{12} \\
(\mathrm{GPa})\end{array}$ & $\begin{array}{l}G_{13} \\
(\mathrm{GPa})\end{array}$ & $\begin{array}{l}G_{23} \\
(\mathrm{GPa})\end{array}$ & $\begin{array}{l}t \\
(\mathrm{~mm})\end{array}$ & $\begin{array}{l}\rho \\
\left(\mathrm{kg} / \mathrm{m}^{3}\right)\end{array}$ \\
\hline half-braid ply & 66.81 & 3.705 & 0.278 & 2.471 & 2.471 & 2.471 & 0.046 & 1480 \\
\hline UD ply & 77.372 & 4.0 & 0.309 & 3.293 & 3.293 & 3.293 & 0.057 & 1000 \\
\hline \hline
\end{tabular}

\section{Dynamic Stability Analysis}

\subsection{Same-sense and Opposite-sense Bending Conventions}

Same-sense bending is defined as occurring when compressive stress is introduced along the BRCs edges; in this case, causing a change of the transverse curvature that is of same sense to the initial transverse curvature of the boom, as shown in Fig. 5 (a). Conversely, opposite-sense bending occurs when the BRC edges are in tension, shown in Fig. 5 (b).

When an extended cantilever BRC is subjected to bending, initially the boom will not fail for sufficiently small accelerations, because the moment is not sufficient to push beyond the linear region into a moment-rotation region with a negative slope (unstable) [27]. As the acceleration increases, the boom starts twisting due to increasing moment and eventually loses its stability. Seffen et al. computationally examined the moment-rotation relationship for tape springs [27] and observed that the applied moment varied approximately linearly with the rotation for small rotations; the tape springs failed at smaller bending moments in same-sense bending compared to opposite-sense bending (see Fig. 6); the failure deformations of the thin-walled carbon fibre reinforced plastic tape hinges subjected to same-sense and opposite-sense bendings were different [28].

\subsection{Conventional Spacecraft Manoeuvrings}

For a conventional spacecraft manoeuvre [30, 18], as illustrated in Fig. 7, can be implemented by applying the maximum angular acceleration, using a single torque actuator located at the centre of the rigid central body, for half of the total manoeuvre time and the 


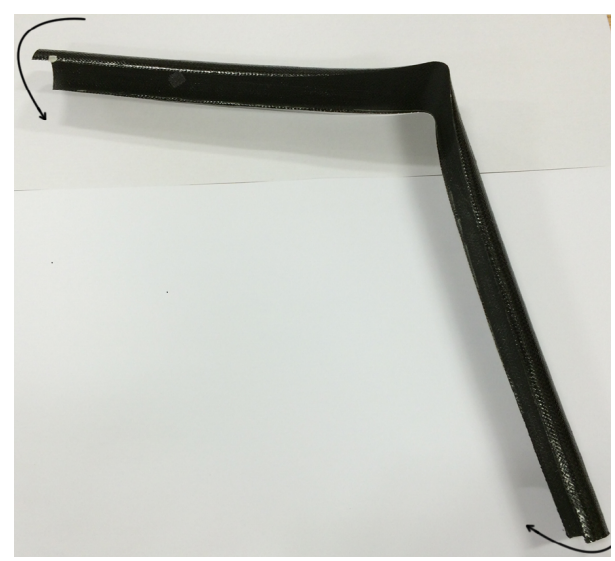

(a) Equal-sense bending

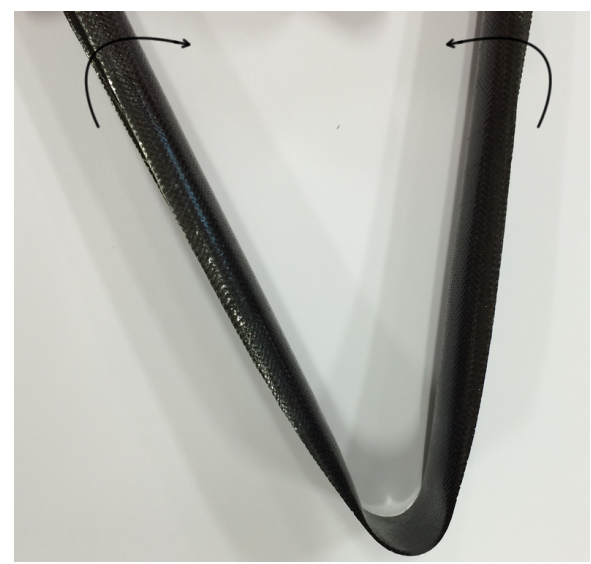

(b) Opposite-sense bending

Fig. 5: BRC same-sense and opposite-sense bending conventions.

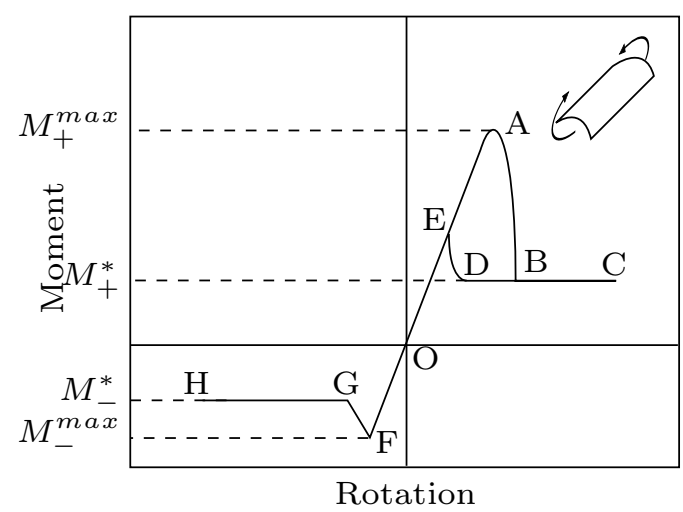

Fig. 6: Schematic moment-rotation relationship for an extended BRC boom [29].

maximum deceleration for the remainder. The conventional acceleration profile, shown in Fig. 7 (a), causes a linear increase and decrease in the manoeuvring rate (see Fig. 7 (b)).

The rest-to-rest manoeuvre is limited by the maximum angular acceleration that can be generated from the torque capability of the spacecraft control authority. Since the manoeuvring rate and position trajectory can be found by integrating the maximum angular acceleration over time, the total time of the manoeuvre $\left(t_{B B}\right)$ is computed using Eq. 1 [19], in which $\theta$ defines the conventional rotation angle, and $a$ is the applied angular acceleration.

$$
t_{B B}=\sqrt{\frac{4 \theta}{a}}
$$

\subsection{FE Modelling Formulation}

In order to study the dynamic performance of extended booms, a spacecraft is simplified as a rigid square plate to which an extended BRC is attached. One end of the BRC boom is rigidly tied to the square plate. An angular acceleration about the $x$ axis or a linear 


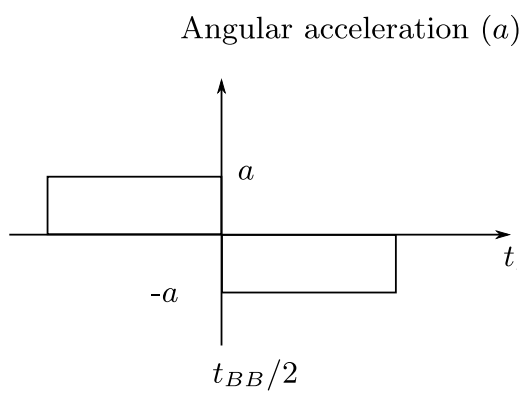

(a)

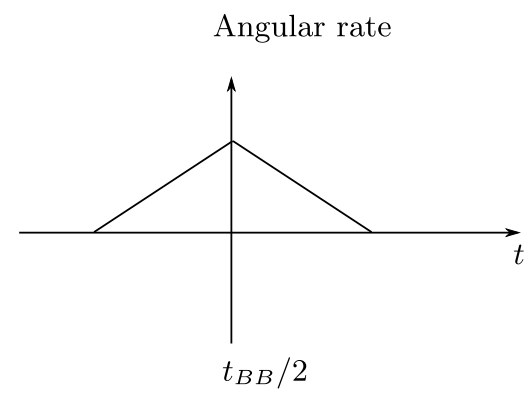

(b)

Rotation $(\theta)$

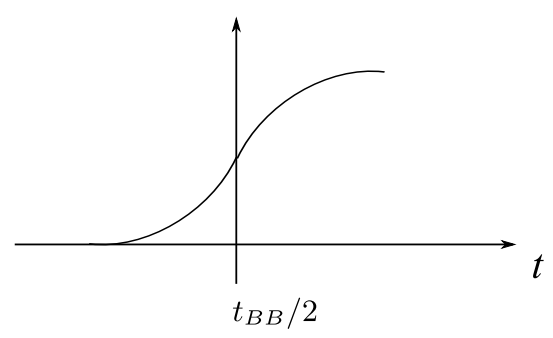

(c)

Fig. 7: Illustration of a conventional spacecraft manoeuvre.

acceleration along the vertical axis of the deployed boom is applied at the centre of the plate to model the boom subjected to bending during a conventional manoeuvre rotating through $90^{\circ}$ or a translation along the $z$ axis (recalling Fig. 3). The material properties of the carbon/epoxy braid ply as given in Tab. 1 are utilized while running the analysis. 4-node shell elements S4R with reduced integration are used to model the composite boom due to their robustness in modelling thin shell structures at a lower computational cost.

\subsubsection{Simulation Techniques}

The extended BRC boom was attached to the rigid plate, and the contact condition defined using a "master-slave" algorithm in ABAQUS Dynamic/Implicit [28]. One end of the boom (slave surface) was tied to the rigid plate (master surface) to define the constraint. The central point of the rigid plate was constrained in all degrees of freedom except rotation about the $x$ axis (see Fig. 3) in the conventional manoeuvre. Two steps were used to model the switch in the conventional angular acceleration profile (recall Fig. 7 (a)). A default automatic time incrementation was selected, to allow the time increment size to be adjusted according to the convergence behaviour. The minimum increment was set to $1 \times 10^{-9} \mathrm{~s}$ and the initial time increment size was defined to $0.0001 \mathrm{~s}$. The other options in the solver were set to default settings.

\section{Results and Experimental Verification}

The dynamic failure of extended single BRCs subjected to root bending is analysed computationally, followed by a validation of a solar array structure shown in Fig. 2 (b) 
and an experimental verification of simulation results for a linear acceleration of $9.8 \mathrm{~m} / \mathrm{s}^{2}$. Finally, a scalability analysis of the boom with respect to its maximum rotational acceleration is complemented.

\subsection{Dynamic Instability of Booms Performing a Rotational Manoeuvre}

The dynamic instability and the maximum angular acceleration $\left(a_{\max }\right)$ of extended BRC booms subjected to same-sense and opposite-sense bending during the conventional manoeuvre are discussed in this section. An angular acceleration profile is implemented at the root of deployed BRC's in FE simulations to simulate bending loads from a host satellite. The Budiansky-Hutchinson criterion [31] for shell structures, which states that "dynamic stability loss occurs when the maximum deflection grows rapidly with the small variation of the load amplitude" is used to estimate the critical angular acceleration that booms can sustain before becoming unstable during a spacecraft's rotational manoeuvre. The crosssection deformation in the transverse direction, $\xi$, is used as the key parameter to estimate the maximum angular acceleration.

The angular acceleration is varied to determine the corresponding maximum cross-section deformations around the region deformed (will be seen in Fig. 9 (b)). For instance, the influence of angular acceleration on the maximum cross-section deformation in the transverse direction for a $2 \mathrm{~m}$ long boom undergoing a $90^{\circ}$ manoeuvre can be seen in Fig. 8 . At the critical acceleration, the maximum cross-section deformation in the transverse direction grows rapidly with a small variation in the angular acceleration, indicating that the boom loses its stability at a critical value of $32 \mathrm{rad} / \mathrm{s}^{2}$ as shown in Fig. 8. Using the same technique the critical accelerations for booms with different lengths can be determined.

The critical angular accelerations of a single boom (B1) and the corresponding solar array structure are estimated to be $4.2 \mathrm{rad} / \mathrm{s}^{2}$ and $4.0 \mathrm{rad} / \mathrm{s}^{2}$ respectively as given in Tab. 2, illustrating the feasibility of analysing the dynamic stability of single booms instead of the solar array structure for the purpose of reducing computing cost. Additionally, the critical accelerations of booms with different lengths and laminate stacks are also given in Tab. 2, in which the $a_{\max }$ values vary with boom length and laminate stacking sequence. For the benchmark boom and boom B2 the $a_{\max }$ values are $1.6 \mathrm{rad} / \mathrm{s}^{2}$ and $2.5 \mathrm{rad} / \mathrm{s}^{2}$ respectively, indicating that the braid angles within the laminate stack have a significant impact on the bending resistance of booms undergoing a conventional manoeuvre; of booms B1 and B2, the shorter one (B1) can withstand a higher angular acceleration.

The stability failures of a benchmark boom under same-sense and opposite-sense bending during spacecraft rotational manoeuvres are illustrated in Fig. 9, in which the boom is deemed to have collapsed because the cross-section had relatively low torsional resistance to the bending moments. The boom subjected to opposite-sense bending can withstand higher bending moments when compared to the one under same-sense bending. 


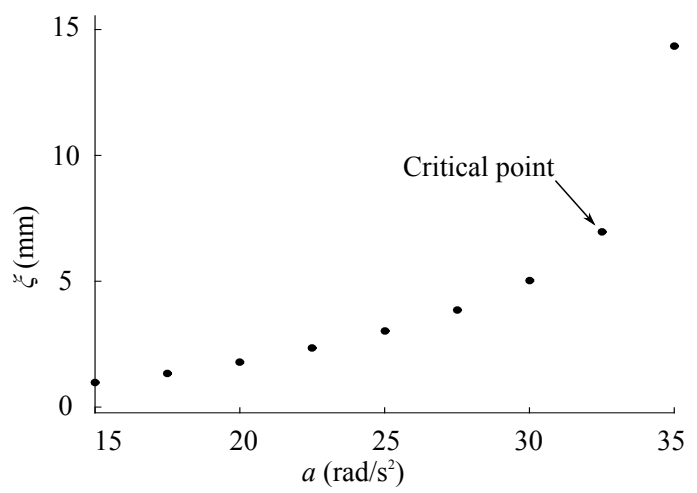

Fig. 8: The maximum cross-section deformation in the transverse direction $\xi$ vs the applied angular acceleration. Results for $L=2 \mathrm{~m}, R=25 \mathrm{~mm}, \beta=180^{\circ}, \theta=\pi / 2 \mathrm{rad}$, and a laminate stacking sequence of $[ \pm 45 / \pm 45 / \pm 45 / \pm 45]$.

Tab. 2: The critical angular accelerations of BRC booms with different lengths and laminate stacks

\begin{tabular}{llllllll}
\hline \hline & & \multicolumn{3}{c}{ BRC boom properties } & \multicolumn{3}{c}{ Braid angles } \\
Boom No. & Stacking sequence & $\mathrm{L}(\mathrm{m})$ & $R$ & $\beta\left(^{\circ}\right)$ & $\alpha_{1}\left(^{\circ}\right)$ & $\alpha_{2}\left(^{\circ}\right)$ & $a_{\max }$ \\
& & & $(\mathrm{mm})$ & & & & $\left(\mathrm{rad} / \mathrm{s}^{2}\right)$ \\
\hline B1 & {$\left[ \pm \alpha_{1} / \pm \alpha_{2} / \pm \alpha_{2} / \pm \alpha_{1}\right]$} & 3 & 38 & 345 & 60 & 26 & 4.2 \\
\hline Solar array & {$\left[ \pm \alpha_{1} / \pm \alpha_{2} / \pm \alpha_{2} / \pm \alpha_{1}\right]$} & 3 & 38 & 345 & 60 & 26 & 4.0 \\
\hline Benchmark boom & {$\left[ \pm \alpha_{1} / \pm \alpha_{2} / \pm \alpha_{2} / \pm \alpha_{1}\right]$} & 5 & 38 & 345 & 45 & 45 & 1.6 \\
\hline B2 & {$\left[ \pm \alpha_{1} / \pm \alpha_{2} / \pm \alpha_{2} / \pm \alpha_{1}\right]$} & 5 & 38 & 345 & 60 & 26 & 2.5 \\
\hline \hline
\end{tabular}

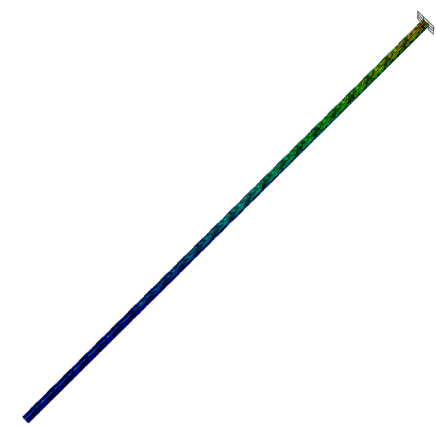

(a) Response of the benchmark boom subjected to small bending moment, results for $a=0.5 \mathrm{rad} / \mathrm{s}^{2}$

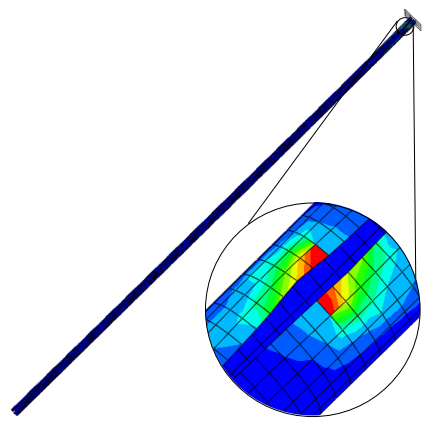

(b) Failure of the benchmark boom subjected to same-sense bending, results for $a=2.5 \mathrm{rad} / \mathrm{s}^{2}$

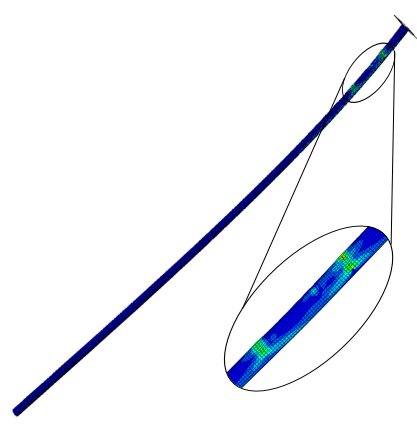

(c) Failure of the benchmark boom subjected to opposite-sense bending, results for $a=40 \mathrm{rad} / \mathrm{s}^{2}$

Fig. 9: Visualisation of the instabilities of benchmark booms subjected to same-sense and opposite-sense bendings. Results for $L=5 \mathrm{~m}, R=38 \mathrm{~mm}, \beta=345^{\circ}$, and a laminate stacking sequence of $[ \pm 60 / \pm 26 / \pm 26 / \pm 60]$. 


\subsection{Experimental Verification}

For the purpose of validating the FE simulation results, an experimental verification was undertaken. While it is extremely difficult to replicate the behaviour of a large flexible lightweight structure in microgravity on the ground, it is possible to simulate the linear acceleration at $9.8 \mathrm{~m} / \mathrm{s}^{2}$ of a cantilevered BRC. Additionally, the simulation results in Sec. 4.1 demonstrated that the booms subjected to same-sense bending were more likely to fail in comparison to opposite-sense bending. The coupling between bending and twisting which occurs in same-sense bending is also not present in opposite-sense bending, making samesense bending the critical case for the majority of applications. Thus, only the same-sense bending experimental tests were performed in this section in order to easily capture the collapse.

A shallow boom with $R=16 \mathrm{~mm}, \beta=180^{\circ}$, and laminate stack of $[ \pm 45 / 0 / \pm 45]$ was initially modelled in ABAQUS. These were the dimensions used on InflateSail [32]. Therefore, the equipment for producing booms with these dimensions was easily accessible in order to set up the experiment. The simulation results for booms under bending are illustrated in Fig. 10. The dynamic failure and critical length of extended BRC booms subjected to samesense bending (see Fig. 10 (a), (b) and (c)) and opposite-sense bending (see Fig. 10 (d), (e) and (f)) induced by linear acceleration along the $z$ axis (recall Fig. 3) were investigated. Again, it was found that the boom subjected to opposite-sense bending can sustain a higher bending moment than it withstood for same-sense bending.

An experiment was set up to determine the point of collapse of this boom under samesense bending due to gravity. A laser distance gauge was utilized to measure the freeend displacement of the boom. The laser provides a contactless method to measure the displacement at the free-end centre of a cantilevered boom with the following procedures: 1) rigidly clamp one end of the test boom to a heavy base; 2 ) hold the boom free end to keep it horizontal; 3) point the laser dot perpendicularly to the centre of the boom free end; 4) release the free end to allow the boom to bend. Several groups of signals were collected for the cantilevered boom with $R=16 \mathrm{~mm}, \beta=180^{\circ}$, linear density of $15 \mathrm{~g} / \mathrm{m}$, and laminate stacking sequence of $[ \pm 45 / 0 / \pm 45]$.

Different lengths of boom were experimentally tested and computationally simulated to visualise the dynamic failure modes when subjected to same-sense bending and to capture the critical length of the boom at collapse. In simulation, a linear acceleration of $9.8 \mathrm{~m} / \mathrm{s}^{2}$ was applied at the centre of the plate as shown in Fig. 11 in the FE model to replicate the effect of gravity on the cantilever boom. The experimental failures and the simulation visualisation results are displayed in Fig. 12, in which the boom twisted when $L=2.3 \mathrm{~m}$ (see Figs. (a) and (c)) and collapsed at $L=2.45 \mathrm{~m}$ as shown in Figs. (b) and (d). The free-end displacements and errors between experiment and simulation of booms with various lengths are given in Tab. 3. The $2.3 \mathrm{~m}$ long cantilevered boom tip vertical displacement was experimentally estimated to be $204 \mathrm{~mm}$, and the simulated value was $216 \mathrm{~mm}$. The difference between the experimental and simulated results was approximately $5.9 \%$. Additionally, the $2.45 \mathrm{~m}$ long BRC boom was used to experimentally and computationally examine the boom collapse under same-sense bending. The correlation with an approximately $5.9 \%$ difference between the experimental and simulated tip displacements and the critical length of the boom collapse subjected to same-sense bending verified reliability of the FE simulations in this loading condition. 


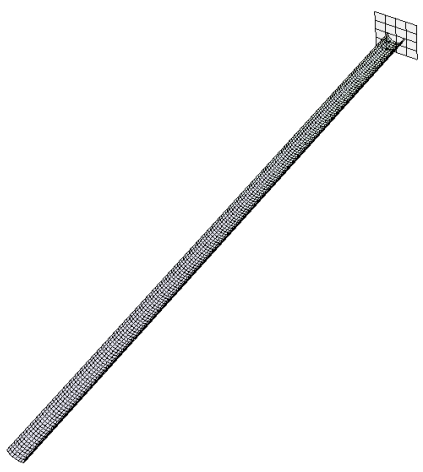

(a) A $1 \mathrm{~m}$ long boom subjected to same-sense bending due to linear acceleration

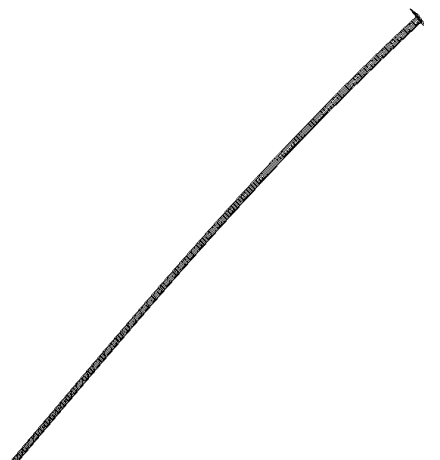

(d) A $3 \mathrm{~m}$ long boom subjected to opposite-sense bending due to linear acceleration

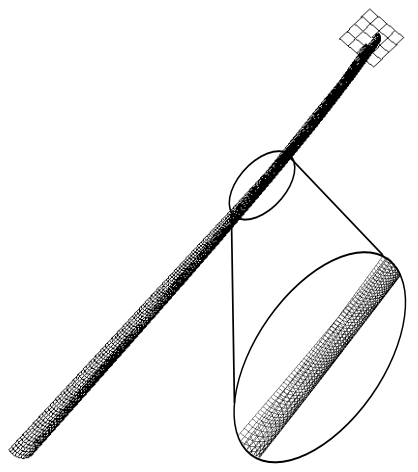

(b) A $2 \mathrm{~m}$ long boom subjected to same-sense bending due to linear acceleration

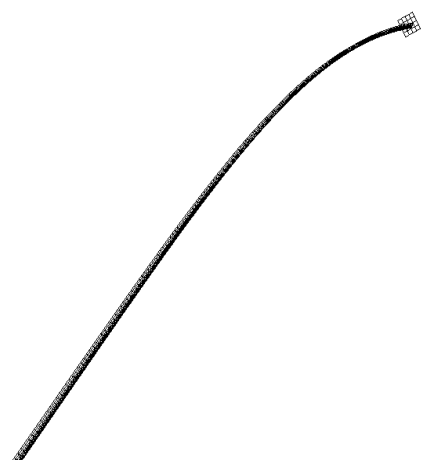

(e) A $5 \mathrm{~m}$ long boom subjected to opposite-sense bending due to linear acceleration

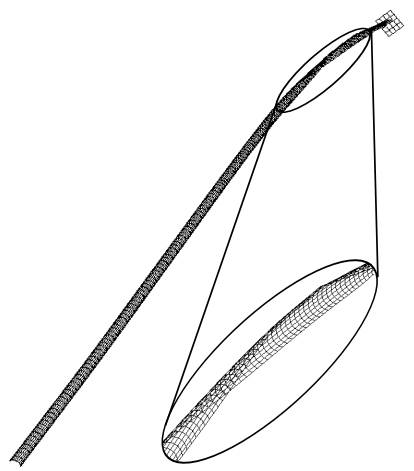

(c) A $3 \mathrm{~m}$ long boom subjected to same-sense bending due to linear acceleration

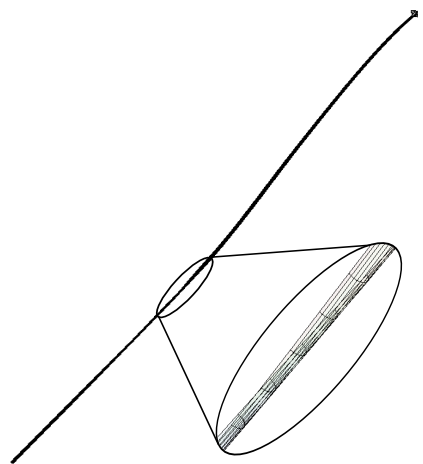

(f) A $7 \mathrm{~m}$ long boom subjected to opposite-sense bending due to linear acceleration

Fig. 10: Simulation of a cantilevered boom subjected to bending due to the linear acceleration of $9.8 \mathrm{~m} / \mathrm{s}^{2}$. Results for $R=16 \mathrm{~mm}, \beta=180^{\circ}$, and a laminate stacking sequence of $[ \pm 45 / 0 / \pm 45]$.

\subsection{Scalability Analysis of BRC Booms}

The deployable "roll-up" PV solar array developed for DeployTech project funded by the European Commission through the Seventh Framework Programme (FP7) was $5 \mathrm{~m}$ long, and $1 \mathrm{~m}$ wide, but it is envisaged that much larger solar arrays can be built. Nevertheless, very long BRCs exhibit low natural frequencies and are prone to failure when subjected to very low accelerations. Additionally, a significant delay in movement at the tip of a cantilevered $\mathrm{BRC}$ or solar array structure appears in extremely long booms, as the effect of a rotation or translation at the root of the boom takes time to propagate along its length. The low natural frequency, instability failure and movement propagation delay result in design limitations of the solar arrays. Furthermore, large displacements due to the movement propagation delay at the tip and the low natural frequencies are limited by the requirements of the attitude control subsystem, which may well be the driving requirement for larger systems. However, only the instability failure limit in the maximum allowable length of a single boom built in a spacecraft performing the conventional manoeuvre (see Fig. 7) is investigated in this section.

In order to achieve a basic understanding of the scalability of the cantilevered booms, 


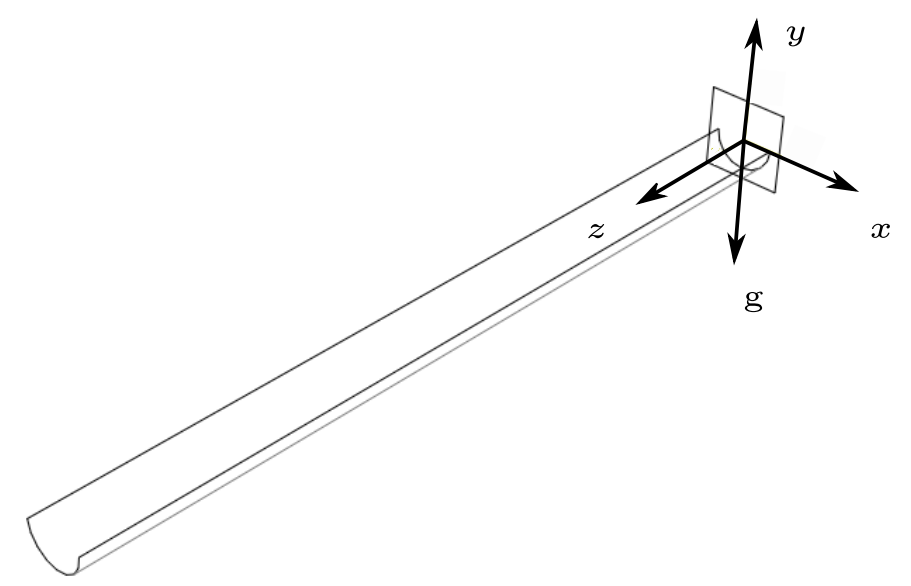

Fig. 11: A cantilever boom model with an acceleration of gravity applied at the plate centre in the FE environment.

Tab. 3: The free-end displacements of BRC booms with different lengths. Results for $R=16 \mathrm{~mm}, \beta=180^{\circ}$, and laminate stacking sequence of $[ \pm 45 / 0 / \pm 45]$.

\begin{tabular}{lll}
\hline \hline \multicolumn{1}{c}{$\mathrm{L}(\mathrm{m})$} & 2.3 & 2.45 \\
\hline Experimental tip displacement $(\mathrm{mm})$ & 204 & Collapse \\
\hline Simulated tip displacement $(\mathrm{mm})$ & 216 & Collapse \\
\hline Error magnitudes $(\%)$ & 5.9 & - \\
\hline \hline
\end{tabular}

and estimate the maximum allowable length of a solar array attached to a satellite performing a conventional manoeuvre, an analysis of the rotational acceleration with respect to the boom length was conducted. The critical rotational acceleration of a single boom can be estimated using the Budiansky-Hutchinson criterion [33]. For instance, the maximum angular accelerations for an extended boom with $R=38 \mathrm{~mm}, \beta=345^{\circ}$, and a laminate layup of $[ \pm 45 / \pm 45 / \pm 45 / \pm 45]$ performing a $90^{\circ}$ conventional manoeuvre in $15 \mathrm{~s}$ [19] (see Fig. 7) are plotted as a function of boom length in Fig. 13. The sustainable angular acceleration is found to decrease approximately with $a_{\max } \propto L^{-3.4}$ as the boom length increases. This finding is useful for predicting the maximum allowable length of a solar array constructed with BRCs. Combining Eq. 1 and $a_{\max } \propto L^{-3.4}$, the estimated maximum length of the boom is approximately $25 \mathrm{~m}$ for a manoeuvrability of $90^{\circ}$ during a conventional manoeuvre in $15 \mathrm{~s}$. In addition, equations available in literature [11] illustrating the rotational acceleration $(\dot{\Omega})$ of a central hub in the dynamic analysis of a rotating cantilever beam were introduced as follows [11]:

$$
\lambda=T^{2} \dot{\Omega}
$$




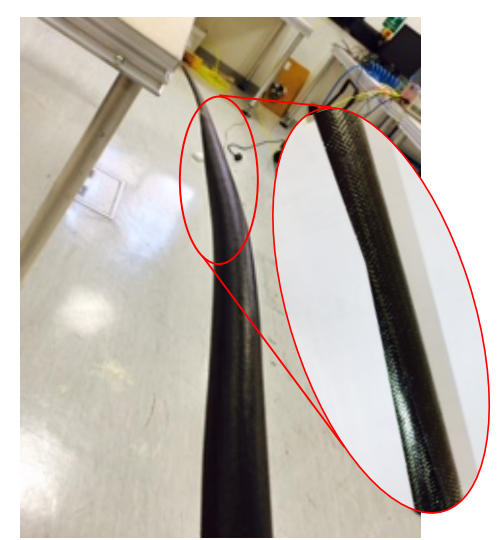

(a) Experimental twisting failure of a $2.3 \mathrm{~m}$ long boom subjected to same-sense bending due to gravity

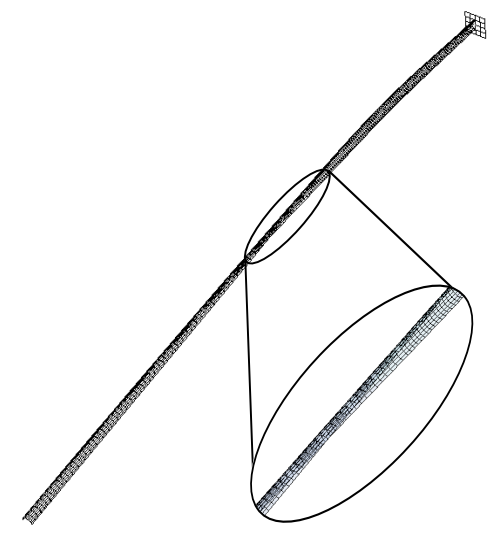

(c) Simulated failure of a $2.3 \mathrm{~m}$ long boom subjected to same-sense bending due to the linear acceleration at the plate centre

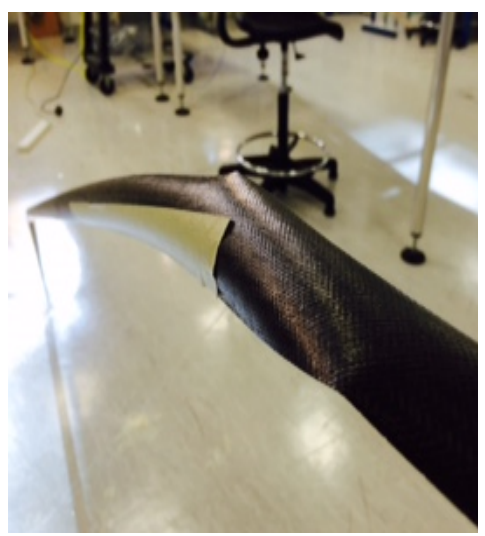

(b) Experimental collapse of a $2.45 \mathrm{~m}$ long boom subjected to same-sense bending due to gravity

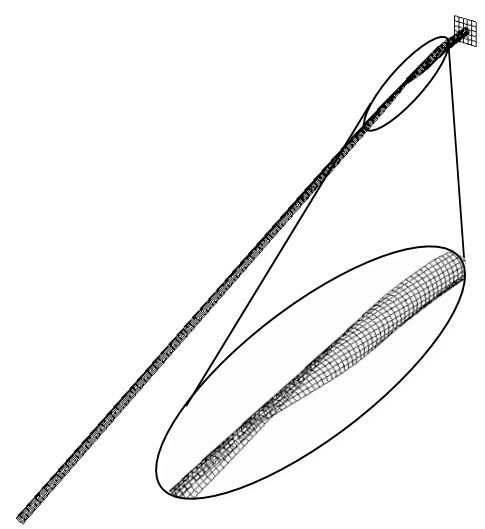

(d) Simulated collapse of a $2.45 \mathrm{~m}$ long boom subjected to same-sense bending due to the linear acceleration at the plate centre

Fig. 12: Experimental and simulation failure modes of a cantilevered boom due to gravity acceleration. Results for $R=16 \mathrm{~mm}, \beta=180^{\circ}$, and a laminate stacking sequence of $[ \pm 45 / 0 / \pm 45]$.

where

$$
T=\sqrt{\frac{\rho A L^{4}}{E I}}
$$

Thus, the rotational acceleration $(\dot{\Omega})$ can be given as:

$$
\dot{\Omega}=\frac{\lambda E I}{\rho A L^{4}}
$$

where $\lambda$ is the dimensionless rotation acceleration of the cantilever beam; $E I$ is the flexural rigidity; $\rho, A$, and $L$ are the mass density, cross-sectional area, and length of the beam. By way of comparison, substituting the dimensions and manoeuvring profile of an identical BRC boom to that described above into Eq. 4, and for $\lambda=1$ when the applied rotation acceleration equals to the maximum angular acceleration, the corresponding boom length is 
approximately $24 \mathrm{~m}$, which is in agreement with the simulation estimation. However, for such a long boom there is a movement propagation delay at the tip meaning that the power law may no longer hold beyond a certain length.

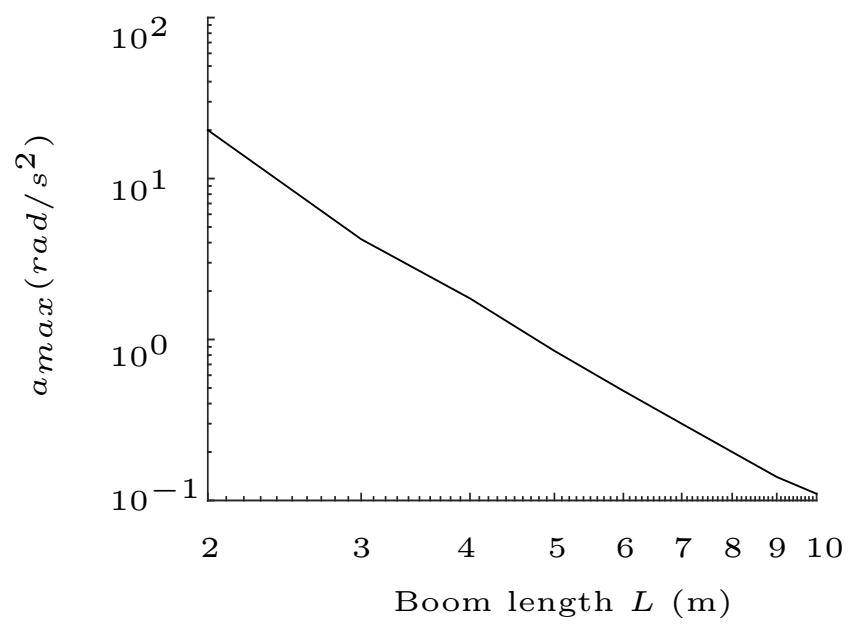

Fig. 13: The maximum angular acceleration of cantilevered BRC booms built into a spacecraft during the conventional manoeuvre as a function of boom length. Results for $R=38 \mathrm{~mm}, \beta=345^{\circ}$, and a laminate layup of $[ \pm 45 / \pm 45 / \pm 45 / \pm 45]$.

\section{Conclusion}

A bending loading condition was applied to modelled BRCs and a solar array built into a simplified spacecraft undergoing a conventional rotational manoeuvre and a translational manoeuvre. The booms can sustain higher opposite-sense bending moments comparing to the ones subjected to same-sense bending. The instability of failure points has been found using the Budiansky-Hutchinson criterion to estimate the maximum angular acceleration that booms can withstand before becoming unstable. The agreement with an approximately $5 \%$ difference between estimated $a_{\max }$ values for a solar array structure and the corresponding single boom illustrates the feasibility of analysing the dynamic stability behaviour of single booms instead of the complete solar array structures. The translational manoeuvre induced from the linear acceleration has been computationally and experimentally investigated. Accordance in the collapse points and failure modes seen in both the simulation and the experiment validate the FE simulations. From the viewpoint of satellite control systems, the coupled rotational and translational manoeuvres about all of the three axes can also be further illustrated.

\section{Acknowledgements}

This work is funded by the Engineering and Physical Sciences Research Council [training grant reference EP/K504580/1]. The authors would like to acknowledge the technical and financial support from RolaTube Technology Ltd. and the European Commission Framework 7 Program project DeployTech (project reference 284474). The authors confirm 
that the data underlying the findings are available without restriction. Details of the data and how to request access are available from University of Surrey publications repository: (http://epubs.surrey.ac.uk).

\section{References}

[1] K. Iqbal, S. Pellegrino, A. Daton-Lovett, Bi-stable composite slit tubes, in: IUTAMIASS Symposium on Deployable Structures: Theory and Applications, Springer, 2000, pp. 153-162.

[2] K. Iqbal, S. Pellegrino, Bi-stable composite shells, in: Proc. 41st AIAA/ASME/ASCE/AHS/ASC Structures, Structural Dynamics, and Materials Conference and Exhibit, Vol. 3, 2000.

[3] A. J. Daton-Lovett, Q. M. Compton-Bishop, R. G. Curry, Deployable structures using bistable reeled composites, in: SPIE 7th Annual International Symposium on Smart Structures and Materials, Vol. 3992, International Society for Optics and Photonics, 2000 .

[4] D. A. Galletly, Modelling the equilibrium and stability of slit tubes, Ph.D. thesis, University of Cambridge (2002).

[5] S. Guest, S. Pellegrino, Analytical models for bistable cylindrical shells, Proceedings of the Royal Society A: Mathematical, Physical and Engineering Science 462 (2006) 839-854.

[6] J. M. Fernandez, Low-cost gossamer systems for solar sailing \& spacecraft deorbiting applications, Ph.D. thesis, University of Surrey (2014).

[7] C. Wu, A. Viquerat, G. Aglietti, Natural frequency optimization and stability analysis of bistable carbon fiber reinforced plastic booms for space applications, in: 3rd AIAA Spacecraft Structures Conference, 2016.

[8] V. Modi, Attitude dynamics of satellites with flexible appendages-a brief review, Journal of Spacecraft and Rockets 11 (1974) 743-751.

[9] G. Benedetti, Derivation of the coupled equations of motion for a beam subjected to three translational accelerations and three rotational accelerations, Tech. rep., Sandia Labs, Livermore, CA (USA) (1979).

[10] T. Kane, R. Ryan, A. Banerjee, Dynamics of a cantilever beam attached to a moving base, Journal of Guidance, Control, and Dynamics 10 (1987) 139-151.

[11] J. Chung, H. H. Yoo, Dynamic analysis of a rotating cantilever beam by using the finite element method, Journal of Sound and Vibration 249 (2002) 147-164.

[12] C. Y. Lin, L. W. Chen, Dynamic stability of rotating pre-twisted blades with a constrained damping layer, Composite Structures 61 (2003) 235-245. 
[13] M. Ganapathi, Dynamic stability characteristics of functionally graded materials shallow spherical shells, Composite Structures 79 (2007) 338-343.

[14] H. Fuji, S. Ishijima, Mission-function control for slew maneuver of a flexible space structure, Journal of Guidance, Control, and Dynamics 12 (1989) 858-865.

[15] W. Ley, K. Wittmann, W. Hallmann, Handbook of space technology, Vol. 22, John Wiley \& Sons, 2009.

[16] M. Sylla, B. Asseke, Dynamics of a rotating flexible and symmetric spacecraft using impedance matrix in terms of the flexible appendages cantilever modes, Multibody System Dynamics 19 (2008) 345-364.

[17] M. Karpenko, S. Bhatt, N. Bedrossian, I. M. Ross, Flight implementation of shortesttime maneuvers for imaging satellites, Journal of Guidance, Control, and Dynamics 37 (2014) 1069-1079.

[18] J. T. King, M. Karpenko, A simple approach for predicting time-optimal slew capability, Acta Astronautica 120 (2016) 159-170.

[19] A. Fleming, P. Sekhavat, I. M. Ross, Minimum-time reorientation of a rigid body, Journal of Guidance, Control, and Dynamics 33 (2010) 160-170.

[20] I. Barkana, J. Z. Ben-Asher, Simple adaptive control applications to large flexible structures, Journal of Guidance, Control, and Dynamics 34 (2011) 1929-1932.

[21] C. Wu, A. Viquerat, Natural frequency optimization of braided bistable carbon/epoxy tubes: analysis of braid angles and stacking sequences, Composite Structures 159 (2017) $528-537$.

[22] Dassault Systèmes, Abaqus 6.14.

URL http://www.3ds.com/products-services/simulia/products/abaqus

[23] A. Viquerat, M. Schenk, M. Straubel, G. Secheli, M. Hillebrandt, M. Shahid, Report on modelling and simulation, EC Framework Programme 7 Deliverable 1.3 for DeployTech (project reference 284474), University of Surrey (2013).

[24] C. Wu, A. Viquerat, G. Aglietti, Improving the natural frequency of bistable carbon fibre reinforced plastic tubes for space applications, Journal of the International Association for Shell and Spatial Structures 56 (2015) 259-267.

[25] E. A. Thornton, Y. A. Kim, Thermally induced bending vibrations of a flexible rolled-up solar array, Journal of Spacecraft and Rockets 30 (1993) 438-448.

[26] A. T. Nettles, Basic mechanics of laminated composite plates (NASA-RP-1351), Tech. rep., NASA Marshall Space Flight Center (1994).

[27] K. A. Seffen, Z. You, S. Pellegrino, Folding and deployment of curved tape springs, International Journal of Mechanical Sciences 42 (2000) 2055-2073. 
[28] J. C. H. Yee, S. Pellegrino, Foldable composite structures, in: Proceedings of IASSAPCS, 2003.

[29] C. Wu, G. Knott, A. Viquerat, Dynamics of curved bistable reeled composites, in: 14th European Conference on Spacecraft Structures, Materials and Environmental Testing, 2016.

[30] J. L. Junkins, J. D. Turner, Optimal spacecraft rotational maneuvers, Elsevier, 2012.

[31] B. Budiansky, Theory of buckling and post-buckling behavior of elastic structures, Advances in Applied Mechanics 14 (1974) 1-65.

[32] A. Viquerat, M. Schenk, B. Sanders, V. Lappas, Inflatable rigidisable mast for endof-life deorbiting system, in: European Conference on Spacecraft Structures, Materials and Environmental Testing (SSMET), 2014.

[33] B. Budiansky, J. W. Hutchinson, Dynamic buckling of imperfection-sensitive structures, in: Applied Mechanics, Springer, 1966, pp. 636-651. 